\title{
A NOTE ON THE NAKAYAMA CONJECTURES
}

\author{
By
}

\author{
R.R. COLBY and K.R. FulleR
}

The Nakayama conjecture states that every finite dimensional algebra of infinite dominant dimension is QF (see [13], [14], [16]). The generalized Nakayama conjecture (GNC) of Auslander and Reiten [2], whose verification would imply Nakayama's conjecture, maintains that the injective envelopes of all the simple modules over an artin algebra $\Lambda$ appear as direct summands of terms in the minimal injective resolution of $\Lambda$; or equivalently, if ${ }_{A} S$ is simple then there is an $i \geqq 0$ such that $\operatorname{Ext}_{\Lambda}^{i}(S, \Lambda) \neq 0$. Jans [12] proved that if $R$ is a left noetherian ring such that the right finitistic global dimension of $R$, $\sup \left\{\operatorname{Pd}\left(N_{R}\right) \mid \operatorname{Pd}\left(N_{R}\right)<\infty\right\}$ (taken over finitely generated right $R$-modules), is finite then if ${ }_{R} M \neq 0$ is finitely generated there is an $i \geqq 0$ with $\operatorname{Ext}_{R}^{i}(M, R) \neq 0$. The finitistic dimension conjecture (arising from questions raised in [3] and [12]), asserts that the finitistic global dimension of every finite dimensional algebra is finite. These conjectures lead us to consider the intermediate condition on finitely generated modules

$$
\operatorname{EXT}_{R}(M, R)=0 \quad \text { implies } \quad M=0
$$

where $\operatorname{EXT}_{R}(M, R)=\bigoplus_{i \geq 0} \operatorname{Ext}_{R}^{i}(M, R)$. We shall say that a ring for which this condition holds satisfies the strong Nakayama conjecture (SNC).

In this note we present a generalization (Theorem 2) of a theorem of Iwanaga [11] by verifying the SNC for a class of rings that properly includes the noetherian rings for which the regular module $R_{R}$ has finite injective dimension. We introduce a condition that appears to be considerably weaker than the ultimately closed condition of [12], [13] and [16]; and we also show (Proposition 4) that it yields the SNC for a class of algebras that contains those for which the Nakayama conjecture was verified by Mueller in [13] and is closely related to those for which the GNC was verified by Auslander and Reiten in [2].

The finitistic dimension conjecture has recently been verified for monomial (or zero-relation) algebras by Green, Kirkman, and Kuzmanovich [9] and by

Received August 21, 1989.

Fuller wishes to express his gratitude for the hospitality of the University of Hawaii during the preparation of this paper. 
Igusa and Zacharia [10]; also we have been informed that Green and Zimmermann-Huisgen have proved it for artin algebras with radical cubed zero. These two classes of algebras bear no apparent relation to the ones for which we verify the SNC.

We begin with a proposition that serves to characterize those left noetherian rings over which the SNC holds in terms of their projective modules.

1. Proposition. Let $R$ be left noetherian. Then $R$ has a non-zero finitely generated left module ${ }_{R} M$ with $\operatorname{EXT}_{R}(M, R)=0$ if and only if there is an exact sequence of finitely generated projective right $R$-modules

$$
0 \longrightarrow Q_{0} \stackrel{k_{1}}{\longrightarrow} Q_{1} \stackrel{k_{2}}{\longrightarrow} Q_{2} \longrightarrow \cdots
$$

such that, letting $L_{i}=$ Coker $k_{i}$

(i) $\operatorname{Ext}_{R}^{1}\left(L_{1}, R\right) \neq 0$ (i.e. $k_{1}$ does not split), and

(ii) $\operatorname{Ext}_{R}^{1}\left(L_{i}, R\right)=0, i \geqq 2$.

Proof: $(\Leftarrow)$ We denote the $R$-dual $\operatorname{Hom}_{R}(-, R)$ by ()$^{*}$. Assume the given conditions and consider the sequence

$$
\cdots \longrightarrow Q_{2}^{*} \stackrel{k_{2}^{*}}{\longrightarrow} Q_{1}^{*} \stackrel{k_{1}^{*}}{\longrightarrow} Q_{0}^{*} \longrightarrow{ }_{R} M \longrightarrow 0 .
$$

where $M=$ Coker $k_{1}^{*} \cong \operatorname{Ext}_{R}^{1}\left(L_{1}, R\right)$. By condition (i) $M \neq 0$ and by condition (ii) the sequence is exact, since, for $i \geqq 1, \operatorname{Ker} k_{i}^{*} / \operatorname{Im} k_{i+1}^{*}=\operatorname{Ext}_{R}^{1}\left(L_{i+1}, R\right)=0$. Now since finitely generated projectives are reflexive and their $R$-duals are projective we see from the commutative diagram

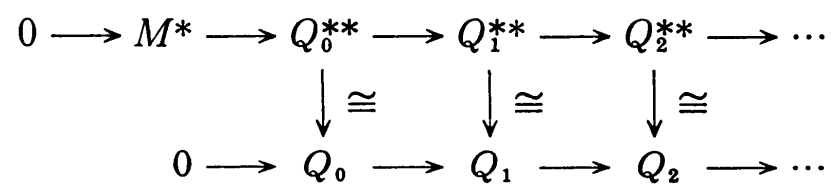

that $\operatorname{EXT}_{R}(M, R)=0$.

$\Leftrightarrow$ Suppose ${ }_{R} M \neq 0$ is finitely generated with $\operatorname{EXT}_{R}(M, R)=0$ and let

$$
\cdots \longrightarrow P_{2} \stackrel{d_{2}}{\longrightarrow} P_{1} \stackrel{d_{1}}{\longrightarrow} P_{0} \stackrel{d_{0}}{\longrightarrow} M \longrightarrow 0
$$

be a projective resolution with all $P_{i}$ finitely generated. Then we get an exact sequence

$$
0 \longrightarrow P_{0}^{*} \stackrel{d_{1}^{*}}{\longrightarrow} P_{1}^{*} \stackrel{d_{2}^{*}}{\longrightarrow} P_{2}^{*} \longrightarrow \cdots
$$

and, moreover, since the $P_{i}$ are reflexive the sequence 


$$
\cdots \longrightarrow P_{2}^{* *} \stackrel{d_{2}^{* *}}{\longrightarrow} P_{1}^{* *} \stackrel{d_{1}^{* *}}{\longrightarrow} P_{0}^{* *}
$$

is exact showing that, if $L_{i}=\operatorname{Coker} d_{i}^{*}, \operatorname{Ext}_{R}^{1}\left(L_{i}, R\right)=0$ for $i \geqq 2$. Also, since $d_{1}^{* *}$ is not epic, $d_{1}^{*}$ is not split monic so $\operatorname{Ext}_{R}^{1}\left(L_{1}, R\right) \neq 0$.

An injective resolution

$$
0 \longrightarrow M \stackrel{\delta_{0}}{\longrightarrow} E_{0} \stackrel{\delta_{1}}{\longrightarrow} E_{1} \stackrel{\delta_{2}}{\longrightarrow} E_{2} \longrightarrow \cdots
$$

of a module $M$ is called ultimately closed (see [12], [13], [16]) in case there is an $n \geqq 1$ such that $\operatorname{Im} \delta_{n}$ has a finite decomposition $\bigoplus_{j=1}^{n} W_{j}$ with each $W_{j}$ isomorphic to a direct summand of some $\operatorname{Im} \delta_{i_{j}}$ with $i_{j}<n$. We shall say that the resolution has a redundant image in case some $\operatorname{Im} \delta_{n}=\bigoplus_{j=1}^{m} W_{j}$ with each $W_{j}$ isomorphic to a direct summand of some $\operatorname{Im} \delta_{i_{j}}$ with $i_{j} \neq n$. The obvious dual notions pertain to projective resolutions.

We note that it follows from [13, Lemma 1] that if $\Lambda$ is an artin algebra and ${ }_{A} M$ is finitely generated, $M$ has an injective resolution of either of tae above types if and only if its minimal injective resolution is of that type. Moreover, it seems to have not been previously remarked that one can easily show in this case that the minimal injective resolution is ultimately closed if and only if the indecomposable modules that appear as direct summands of the various $\operatorname{Im} \delta_{i}$ form a finite set-namely those that appear in the $\operatorname{Im} \delta_{i}$, with $0 \leqq i \leqq n-1$. In particular, for ${ }_{A} M$ to have an ultimately closed injective resolution it is necessary that each indecomposable direct summand of $M$ have one; not so for a redundant image. Indeed, an injective resolution of $M \oplus \operatorname{Im} \delta_{i}$ has a redundant image for any $i \geqq 1$.

Our generalization of Iwanaga's [11, Theorem 2] follows. We note that his proof suffices if $R_{R}$ has finite injective dimension, and our result (as well as Proposition 4 and Corollary 5) is new under the intermediate hypothesis of an ultimately closed injective resolution.

2. THEOREM. Let $R$ be left noetherian and suppose that the right regular module $R_{R}$ has an injective resolution with a redundant image. If $\operatorname{EXT}_{R}(M, R)=0$ with ${ }_{R} M$ finitely generated, then $M=0$, i.e., the SNC is true for left $R$-modules.

PROOF: Suppose there is an exact sequence of finitely generated projective right $R$-modules

$$
0 \longrightarrow Q_{0} \stackrel{k_{1}}{\longrightarrow} Q_{1} \stackrel{k_{2}}{\longrightarrow} Q_{2} \stackrel{k_{3}}{\longrightarrow} \cdots
$$

with $L_{i}=\operatorname{Coker} k_{i}$, such that $\operatorname{Ext}_{R}^{1}\left(L_{i}, R\right)=0, i \geqq 2$. Then since the sequence is 
exact, $\operatorname{Ext}_{R}^{j}\left(L_{i},-\right)=0$ for $j>i$; and if $1 \leqq j<i$,

$$
\operatorname{Ext}_{R}^{j}\left(L_{i}, R\right)=\operatorname{Ext}_{R}^{1}\left(L_{i-j+1}, R\right)=0
$$

since $i-j+1 \geqq 2$. Thus $\operatorname{Ext}_{R}^{j}\left(L_{i}, R\right)=0$ if $1 \leqq j \neq i$. Now suppose $n \geqq 1$ and

$$
0 \longrightarrow R_{R} \longrightarrow E_{0} \stackrel{\delta_{1}}{\longrightarrow} E_{1} \stackrel{\delta_{2}}{\longrightarrow} E_{2} \longrightarrow \cdots
$$

is an injective resolution with $\operatorname{Im} \delta_{n}=\bigoplus_{j} W_{j}$ and $W_{j}$ a direct summand of $\operatorname{Im} \delta_{i_{j}}$ for $i_{j} \neq n$. Then by Proposition 1 we need only show that $\operatorname{Ext}_{R}^{1}\left(L_{1}, R\right)=0$. But

$$
\begin{aligned}
\operatorname{Ext}_{R}^{1}\left(L_{1}, R\right) & =\operatorname{Ext}_{R}^{n+1}\left(L_{n+1}, R\right) \\
& =\operatorname{Ext}_{R}^{1}\left(L_{n+1}, I_{n}\right) \\
& \cong \bigoplus_{j} \operatorname{Ext}_{R}^{1}\left(L_{n+1}, W_{j}\right)
\end{aligned}
$$

and $\operatorname{Ext}_{R}^{1}\left(L_{n+1}, W_{j}\right)$ is a direct summand of

$$
\operatorname{Ext}_{R}^{1}\left(L_{n+1}, \operatorname{Im} \delta_{i_{j}}\right)=\operatorname{Ext}_{R}^{i j^{+1}}\left(L_{n+1}, R\right)=0 .
$$

In [2] Auslander and Reiten proved that for artin algebras the GNC is equivalent to the conjecture that a finitely generated generator ${ }_{A} G$ over an artin algebra $\Lambda$ is projective if $\operatorname{Ext}_{\Lambda}^{i}(G, G)=0$ for all $i \geqq 1$. Moreover they proved that if ${ }_{A} G$ has an ultimately closed projective resolution and $\operatorname{Ext}_{A}^{i}(G, G)$ $=0$ for all $i \geqq 1$, then ${ }_{A} G$ is projective. One easily checks that, in fact, their proof of the latter result only requires that the projective resolution has a redundant kernel. Employing the following lemma, versions of which are contained in [2] and [13], we shall apply Theorem 2 to show that if ${ }_{A} G$ has an injective resolution with a redundant image and $\operatorname{Ext}_{A}^{i}(G, G)=0$, for all $i \geqq 1$, then the SNC holds for right $\operatorname{End}\left({ }_{A} G\right)$-modules.

In the cases to be considered, $E$-dom. $\operatorname{dim} . M \geqq n$ just means that the first $n$ terms $E_{0}, \cdots, E_{n-1}$ in an injective resolution of $M$ are direct sums of direct summands of $E$, i. e., belong to $\operatorname{Add} E$ (see [16]); and $E(M)$ denotes the injective envelope of $M$.

3. LEMMA. If $R$ is an artinian ring with radical $J, e$ is an idempotent in $R$ and $E=E(R e / J e)$, then for any $n \geqq 0, E$-dom. dim. $R \geqq n+2$ if and only if $e R$ is a faithful and balanced right $R$-dodule and $\operatorname{Ext}_{e R e}^{i}(e R, e R)=0$ whenever $0<i \leqq n$.

ProOF : $(\Leftarrow)$ According to [5, Lemma 2.2] every indecomposable injective module over $e R e$ is of the form $e X$ where $X$ is an indecomposable direct summand of ${ }_{R} E$. Thus the minimal injective resolution of $e R$ is of the form 


$$
0 \longrightarrow{ }_{e R e} e R \longrightarrow e E_{0} \longrightarrow e E_{1} \longrightarrow \cdots
$$

with $E_{i} \in \operatorname{Add} E$. Now the left exactness of $\operatorname{Hom}_{e R e}(e R,-)$ and our assumption that $\operatorname{Ext}_{e R e}^{i}(e R, e R)=0$ for $0<i \leqq n$ implies exactness up to $n$ in

$0 \longrightarrow \operatorname{Hom}_{e R e}(e R, e R) \longrightarrow \operatorname{Hom}_{e R e}\left(e R, e E_{0}\right) \longrightarrow \operatorname{Hom}_{e R e}\left(e R, e E_{1}\right) \longrightarrow \cdots$

so since $e R_{R}$ is faithful and balanced and

$$
E_{i} \cong \operatorname{Hom}_{R}\left(R, E_{i}\right) \cong \operatorname{Hom}_{e R e}\left(e R, e E_{i}\right)
$$

by $\left[5\right.$, Lemma 2.1], we obtain an injective resolution of $R$ with $E_{0}, E_{1}, \cdots, E_{n+1}$ in Add- $E$.

$(\Rightarrow)$ Assuming the hypothesis, given the injective resolution

$$
0 \longrightarrow{ }_{R} R \longrightarrow E_{0} \longrightarrow E_{1} \longrightarrow \cdots
$$

with $E_{0}, E_{1}, \cdots, E_{n+1}$ in Add- $E, e R_{R}$ is faithful and balanced by [6, Theorem 4], and we have an exact sequence

$$
0 \longrightarrow e R \longrightarrow e E_{0} \longrightarrow e E_{1} \longrightarrow \cdots \longrightarrow e E_{n+1}
$$

where the $e E_{i}, i=1, \cdots, n+1$ are injective by [5, Lemma 2.2]. Now since the isomorphisms $E_{i} \cong \operatorname{Hom}_{e R e}\left(e R, e E_{i}\right)$ are natural, the exactness of the bottom row of the diagram

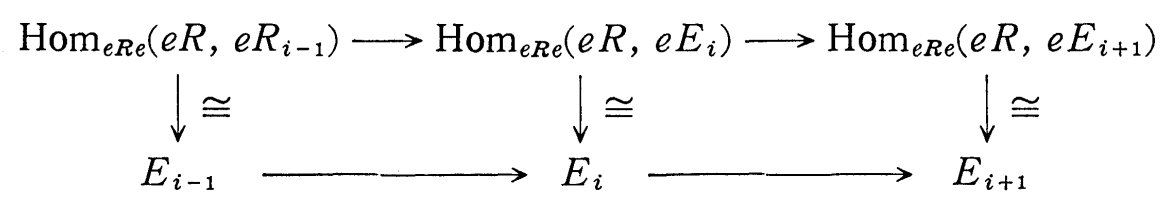

implies that $\operatorname{Ext}_{e R e}^{i}(e R, e R)=0, i=1, \cdots, n$.

It follows as in Auslander and Reiten [2] that if $e$ is an idempotent in an artinian ring $R$ such that $R e / J e$ consists precisely of the simples ${ }_{R} S$ with $\operatorname{EXT}_{R}(S, R) \neq 0$ and $e R e e R$ has a projective resolution with a redundant kernel, then $R$ satisfies the GNC for left modules. It seems not to be known, even for algebras, whether the GNC on one side implies it on the other, but regarding right $R$-modules we have

4. Proposition. Let $e$ be an idempotent in an artinian ring $R$ such that the $E(R e / J e)$-dominant dimension of $R$ is infinite. If $e_{R e} e R$ has an injective resolution with a redundant image then the strong Nakayama conjecture is true for right $R$-modules.

Proof : Let

$$
0 \longrightarrow{ }_{e R e} e R \stackrel{d_{0}}{\longrightarrow} F_{0} \stackrel{d_{1}}{\longrightarrow} \dot{F}_{1} \stackrel{d_{2}}{\longrightarrow} \cdots
$$


be an injective resolution of $e R$ over $e R e$ such that, letting $K_{i}=\operatorname{Im} d_{i}, K_{n}=\oplus_{j} V_{j}$ with $V_{j}$ a direct summand of $K_{i_{j}}, i_{j} \neq n$. Then Lemma 3 shows that the induced sequence

$$
0 \longrightarrow{ }_{R} R \stackrel{\delta_{0}}{\longrightarrow} \operatorname{Hom}_{e R e}\left(e R, F_{0}\right) \stackrel{\delta_{1}}{\longrightarrow} \operatorname{Hom}_{e R e}\left(e R, F_{1}\right) \longrightarrow \cdots
$$

is exact and, moreover, it follows that we can identify $\operatorname{Im} \delta_{i}$ with $\operatorname{Hom}_{e R e}\left(e R, K_{i}\right)$ for $i \geqq 0$. Thus this is an injective resolution of ${ }_{R} R$ (see [1, Exercise 20.8]), and $\operatorname{Im} \delta_{n} \cong \oplus_{j} \operatorname{Hom}_{e R e}\left(e R, V_{j}\right)$ is redundant. Now the proposition follows from Theorem 2.

If ${ }_{\Lambda} G$ is a finitely generated generator over an artin algebra $\Lambda$ and $R=$ $\operatorname{End}\left({ }_{1} G\right)$ then there is an idempotent $e \in R$ and a Morita equivalence $F: \Lambda$-Mod $\rightarrow e R e$-Mod such that $F(G) \cong{ }_{e R e} e R$. Thus from Lemma 3 and Proposition 4 we have

5. COROLlary. Let ${ }_{A} G$ be a finitely generated generator over an artin algebra $\Lambda$ and suppose that ${ }_{\Lambda} G$ has an injective resolution with a redundant image. If $\operatorname{Ext}_{\Lambda}^{i}(G, G)=0$ for $i=1,2, \cdots$, then the strong Nakayama conjecture is true for right $\operatorname{End}\left({ }_{\Lambda} G\right)$-modules.

An artinian ring $R$ has dominant dimension $\geqq n>0$ in case it is QF-3 with minimal faithful modules $R f$ and $e R$ such that $R f$-dom. $\operatorname{dim} . R \geqq n$; then $R f \cong$ $E(R e / \mathrm{Je})$ (see [5] and [16]). A well known result of Mueller [13] (see also [16, Theorem 8.3]) states that if $e R e R$ has an ultimately closed injective resolution then Nakayama's conjecture is true for $R$; it follows from Proposition 4 that a redundant image is sufficient.

6. COROLLARY. Nakayama's conjecture is true for an artinian QF-3 ring $R$ with minimal faithful right module $e R$ such that $e R e R$ has an injective resolution with a redundant image.

7. Remarks: (a) According to Mueller [13, Theorem 9], if ${ }_{\Lambda} G$ is a finitely generated generator-cogenerator over an algebra $\Lambda$ such that ${ }_{\Lambda} G$ has an ultimately closed injective resolution then Nakayama's conjecture is true for $\operatorname{End}\left({ }_{A} G\right)$. It follows from Corollary 6 (see [16, Theorems 5.1 and 5.2]) that, even if the minimal injective resolution

$$
0 \longrightarrow{ }_{\Lambda} G \stackrel{\delta_{0}}{\longrightarrow} E_{0} \stackrel{\delta_{1}}{\longrightarrow} E_{1} \longrightarrow \cdots
$$

is not ultimately closed, Nakayama's conjecture is true for $\operatorname{End}\left({ }_{A}\left(G \oplus \operatorname{Im} d_{i}\right)\right)$ whenever $i \geqq 1$. 
(b) An $R$-module $M$ is completely torsion in case $\operatorname{EXT}_{R}(M, R)=0$. Thus the SNC holds for a ring in case it has no finitely generated completely torsion modules other than 0 . On the other hand, considering arbitrary modules, Nunke's Theorem [15] shows that there are no non-zero completely torsion abelian groups; while over a simple self injective ring (see [8] for a nonartinian example) every non-generator is completely torsion. One wonders exactly which rings have no non-trivial completely torsion modules.

(c) Regarding this last question, if $R$ is a left artinian ring with radical $J$ and $P_{i} \stackrel{d_{i}}{\rightarrow} P_{i-1}$ is a map between arbitrary non-zero projective left $R$-modules such that $\operatorname{Im} d_{i} \leqq J P_{i-1}$, then $P_{i}^{*}$ and $P_{i-1}^{*}$ are projective and non-zero (since products of projective right $R$-modules are projective $[1$, Theorems 19.20 and 28.4]) and $\operatorname{Im} d_{i}^{*} \subseteq \operatorname{Hom}_{R}\left(P_{i}, J\right)=P_{i}^{*} J$ (since ${ }_{R} J$ is finitely generated). It follows that, if ${ }_{R} M \neq 0$ is completely torsion with minimal projective resolution

$$
\cdots \longrightarrow P_{2} \stackrel{d_{2}}{\longrightarrow} P_{1} \stackrel{d_{1}}{\longrightarrow} P_{0} \longrightarrow M \longrightarrow 0
$$

then in the exact sequence

$$
0 \longrightarrow P_{0}^{*} \stackrel{d_{1}^{*}}{\longrightarrow} P_{1}^{*} \stackrel{d_{2}^{*}}{\longrightarrow} P_{2}^{*} \longrightarrow \cdots
$$

$\operatorname{Im} d_{2}^{*}$ is not projective, so $\operatorname{Pd}\left(\operatorname{Im} d_{i+1}^{*}\right)=i$ for $i=1,2, \cdots$. Thus if the finitistic global dimension (taken over all right $R$-modules) of a left artinian ring $R$ is finite, then $R$ has no non-zero completely torsion left modules. (c.f., Jans [12, Corollary 3.2] pertaining to finitely generated modules.)

(d) It is not difficult to show that the torsionless $L_{2}, L_{3}, \cdots$ of Proposition 1 are actually reflexive. It follows that the SNC is true for the algebras described in the concluding remark (a) of [4].

We conclude with examples of an algebra whose minimal injective resolution has a redundant image but is not ultimately closed, and one whose minimal injective resolution does not even have a redundant image.

8. EXAMPLES: We employ the notation and results for diagram algebras and algebra semigroups given in $[7$, Section 1$]$.

(a) Let $K$ be a field and let $R=K \mathscr{R}$ where $\mathscr{R}$ is the algebra semigroup with diagram $\mathscr{R}_{l}=\mathscr{P} \cup Q$

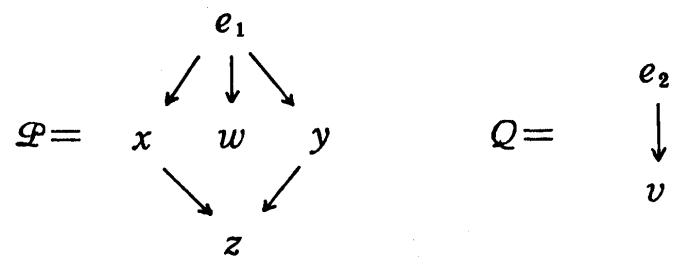


with non-zero products $x y=y x=z, e_{i}^{2}=e_{i}, e_{2} w e_{1}=w, e_{1} v e_{2}=v$ and $e_{1} u e_{1}=u$ for all other nodes $u$. Then ${ }_{R} R=P \oplus Q$ with $P=K \mathscr{Q}=R e_{1}$ and $Q=K Q=R e_{2}$. Moreover, since the cancellation condition of $[7$, Section 1$]$ is satisfied, these and the diagram $R_{r}$ for $R_{R}$ are strong diagrams, and if $S_{i}=R e_{i} / J e_{i}$ then

$$
E\left(S_{1}\right)=U=K q \text { and } E\left(S_{2}\right)=V=K C V
$$

where $\mathcal{U}$ and $\mathcal{V}$ are left $\mathscr{R}$-modules with diagrams

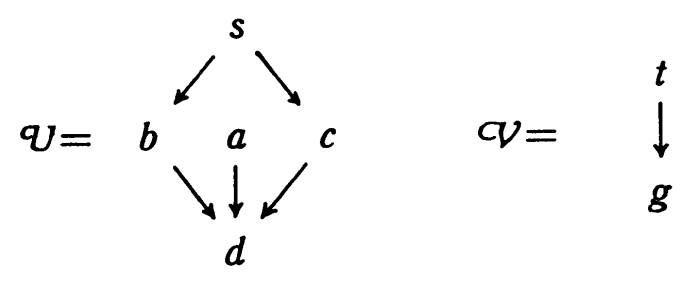

with non-zero $R$-scalar products $x s=b, y s=c, y b=v a=x c=d, e_{2} a=a, e_{2} g=g$ and $e_{1} m=m$ for all other nodes $m$ in $\cup \cup \mathcal{C}$. Now, for $k=1,2, \cdots$, let

$$
M_{k}=P_{1} \oplus \cdots \oplus P_{k} / L
$$

where $P \cong P_{i}$ via $u \mapsto u_{i}$ and $L$ is spanned by

$$
\left\{w_{i}, z_{i} \mid i=1, \cdots, k\right\} \cup\left\{(-1)^{j+1}\left(y_{j}-x_{j+1}\right) \mid j=1, \cdots, k-1\right\} .
$$

Then

$$
M_{1} \cong P / \operatorname{Soc} P \cong E / R a
$$

and each $M_{k}$ is indecomposable being, for example, an interlacing of $k$ copies of $M_{1}$ in the sense of [17]. Now one easily checks that

$$
h: P_{1} \oplus \cdots \oplus P_{k} \longrightarrow U_{1} \oplus \cdots \oplus U_{k+1}
$$

via

$$
h: \sum_{i=1}^{k} p_{i} \longmapsto \sum_{i=1}^{k}(-1)^{k+1} p_{i}\left(c_{i}-b_{i+1}\right)
$$

(where $U \cong U_{i}$ via $u \mapsto u_{i}$ ) has

$$
\operatorname{Ker} h=L \text { and } \quad \text { Coker } h \cong M_{k+1} \oplus S_{2}^{k+1} \text {, }
$$

and thus $h$ induces an exact sequence

$$
0 \longrightarrow M_{k} \longrightarrow E\left(M_{k}\right) \longrightarrow M_{k+1} \oplus S_{2}^{k+1} \longrightarrow 0 .
$$

Finally, we observe that in the minimal injective resolution

$$
0 \longrightarrow{ }_{R} R \longrightarrow E_{0} \stackrel{\delta_{1}}{\longrightarrow} E_{1} \stackrel{\delta_{2}}{\longrightarrow} E_{2} \longrightarrow \cdots
$$

we have

$$
\operatorname{Im} \delta_{1} \cong S_{1} \oplus S_{2} \oplus M_{1}
$$


and

$$
\begin{aligned}
\operatorname{Im} \delta_{2} & \cong U / S_{1} \oplus V / S_{2} \oplus E\left(M_{1}\right) / M_{1} \\
& \cong M_{1} \oplus S_{2} \oplus S_{1} \oplus M_{2} \oplus S_{2}^{2}
\end{aligned}
$$

so that $\operatorname{Im} \delta_{1}$ is redundant; and by (\#) it follows inductively that the resolution is not ultmately closed.

(b) For the second example, $K v$ is an ideal in $R$ and an argument similar to that just given shows that the minimal injective resolution of $R / K v$ has no redundant image. In fact $\operatorname{Im} \delta_{1} \cong S_{1}^{2}$ and $\operatorname{Im} \delta_{k} \cong M_{k-1}^{2}, k=2,3, \cdots$ (This example answers a question suggested to us by T. Wakamatsu.)

Addedinproof : In his paper Duality in Noetherian rings, Proc. Amer. Math. Soc. 12 (1961), 829-835, Jans referred to rings with no completely torsion modules assatisfying Nunke's condition.

\section{References}

[1] Anderson, F.W. and Fuller, K.R., "Rings and Categories of Modules", SpringerVerlag, Inc., New York, 1974.

[2] Auslander, M. and Reiten, I,, On a generalized version of the Nakayama conjecture, Proc. Amer. Math. Soc. 52 (1975), 69-74.

[3] Bass, H., Finitistic dimension and a homological generalization of of semiprimary rings, Trans. Amer. Math. Soc. 95 (1960), 466-488.

[4] Colby, R.R., Nakayama's conjecture and the double dual functors, J. Algebra 94 (1985), 546-557.

[5] Fuller, K.R., On indecomposable injectives over artinian rings, Trans. Amer. Math. Soc. 29 (1969), 115-135.

[6] Fuller, K.R., Double centralizers of injectives and projectives over artinian rings, Illinois J. Math. 14 (1970), 658-664.

[7] Fuller, K.R. and Ohtake, K., Strong module diagrams and Frobenius diagram algebras, Comm. in Algebra 17 (1989), 259-298.

[8] Goodearl, K.R., Simple self-injective rings need not be artinian, Comm. Algebra 2 (1974), 83-89.

[9] Green, E.L., Kirkman, E. and Kuzmanovich, J., Finitistic dimensions of finite dimensional monomial algebras, preprint.

[10] Igusa, K. and Zacharia, D., Syzygy pairs in a monomial algebra, preprint.

[11] Iwanaga, Y., On rings with finite self-injective dimension, Comm. Algebra 7 (1979), 393-414.

[12] Jans, J.P., Some generalizations of finite projective dimension, Illinois J. Małh. 5 (1961), 334-344.

[13] Mueller, B. J., The classification of algebras by dominant dimension, Can. J. Math. 20 (1968), 398-409.

[14] Nakayama, T., On algebras with complete homology, Abh. Math. Sem. Univ. Hamburg 22 (1958), 300-307.

[15] Nunke, R. J., Modules of extensions over Dedekind rings, Illinois J. Math. 3 (1959), 222-241. 
[16] Tachikawa, H., "Quasi Frobenius rings and generalizations", Springer LNM 351, Springer-Verlag, Inc., Berlin and New York, 1973.

[17] Tachikawa, H., A note on algebras of unbounded representation type, Proc. Japan Acad. 36 (1960), 59-61.

Department of Mathematics, University of Hawaii, Honolulu,

HI 96822 U.S. A.

Department of Mathematics, University of Iowa, Iowa City,

IA 52242 U.S.A. 